\title{
Jitter Limitations on Multi-Carrier Modulation
}

\author{
J.H.R. Schrader, E.A.M. Klumperink, B. Nauta \\ IC Design Group, MESA+ Research Inst., Univ. of Twente \\ Enschede, The Netherlands \\ E-mail: j.h.r.schrader@utwente.nl
}

\author{
J.L. Visschers \\ NIKHEF \\ Amsterdam, The Netherlands
}

\begin{abstract}
A feasibility study is made of an OFDM system based on analog multipliers and integrate-and-dump blocks, targeted at $\mathbf{G b} / \mathbf{s}$ copper interconnects. The effective amplitude variation of the integrator output caused by jitter is explained in an intuitive way by introducing correlation plots. For a given rms jitter and error rate, high frequency carriers allow for less modulation depth than low frequency carriers. A jitter limit on the total system bit rate is calculated, which is a function of rms jitter, bandwidth, and specified system symbol error rate. It is concluded that, because of the high sensitivity to timing errors inherent to OFDM, traditional PAM systems with equal bandwidth and error rate are more feasible.
\end{abstract}

\section{INTRODUCTION}

There is a continuous demand for higher bit rate in shortrange wireline and PCB communication. The traditional way of transmitting data over short length copper wires is to use Pulse Amplitude Modulation (PAM), e.g. [1]. However, the spectral efficiency $(\mathrm{bps} / \mathrm{Hz})$ of such systems is much lower than that of Digital Subscriber Line (DSL) modem techniques. Furthermore, these techniques might be interesting when the channel transfer contains spectral nulls (PCB tracks and connectors). We investigate the possibility of extending the bandwidth of Orthogonal Frequency Division Multiplexing (OFDM) techniques to the $\mathrm{GHz}$ order. In common Discrete Multi-Tone (DMT) implementations the ADCs and DACs and the complex digital processing put limits on the maximum bandwidth. A possible way to overcome the bandwidth limitation is to use analog multipliers and integrate-and-dump blocks. We will study the system shown in fig. 1.

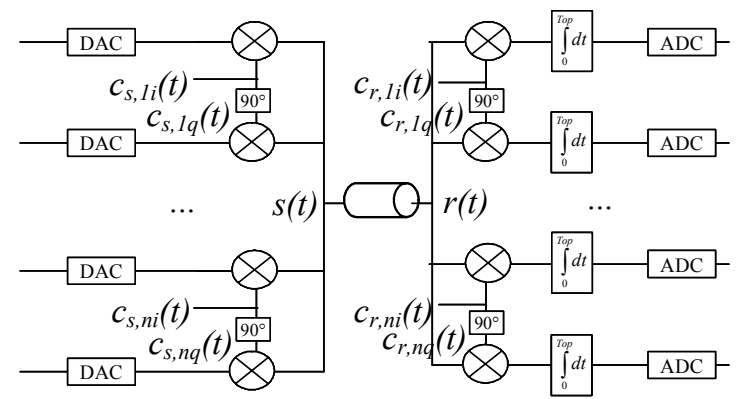

Fig. 1. Multi-carrier system using analog correlation
Parallelization is used for the $\mathrm{AD} / \mathrm{DA}$-converters and integrate-and-dump blocks, which relaxes bandwidth requirements. As with any OFDM system, this system transmits and receives symbols that contain multiple tones (where 'tone' is defined as a single carrier frequency). Both the in-phase and quadrature component of a tone are modulated with data. At the transmitter side the in-phase and quadrature carrier signals $c_{s, x i}(t)$ and $c_{s, x q}(t)(x=\{1, n\})$ are multiplied by the data and the result added in $s(t)$. At the receiver, correlation is implemented with a multiplier and an integrate-and-dump block. The signal $r(t)$ is demodulated using locally generated carriers $c_{r, x q}(t)$ and $c_{r, x i}(t)(x=\{1, n\})$. The system choice may seem arbitrary but is well suited for analysis of jitter effects.

Jitter is expected to have a large impact on the system. In this paper the jitter limitation on the system bit rate will be calculated for a certain specified symbol error rate, and compared to a PAM system. Jitter analyses have been performed in e.g. [2], which is a thorough mathematical analysis of jitter effects on DMT systems. However, [2] is based on a standard DMT system and furthermore we feel that the purely mathematical approach lacks intuitive insight to help designers analyze and improve their systems. The correlation plot based analysis in this paper presents an intuitive way of understanding the mechanism of effective amplitude variation of the integrator output caused by jitter. It serves to improve understanding of jitter impact on system bit rate limits and to draw conclusions about the feasibility of such a system.

\section{JITTER ANALYSIS}

\section{A. Orthogonality loss caused by jitter}

The transmitted data is modulated on several orthogonal carriers. In order to avoid interference these have to comply with the orthogonality constraint, which is defined as

$$
\int_{0}^{T o p} c_{n}(t) \cdot c_{m}(t) d t=0 \quad n \neq m .
$$

where $c_{n, m}(t)$ are carriers and $T_{o p}$ is the length of the receiver integration period ('orthogonality period'). Candidates for tone frequencies $f_{c}$ are harmonic frequencies 
$n / T_{o p}$. Integration over exactly $T_{o p}$ delivers perfectly orthogonal carriers.

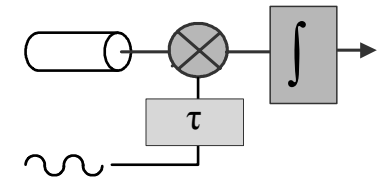

Fig. 2. Influence of delay $\tau$ on correlator receiver output

The analysis goal is to determine the change in the integrator output as a function of variations in $\tau$ (time shift between transmitter and receiver), as illustrated in fig. 2 . Time offsets over the symbol, caused by jitter, lead to imperfect separation of the in-phase and quadrature component of the carrier. We will analyze crosstalk between the in-phase and quadrature components of a carrier at a given frequency.

\section{B. Definitions}

The receiver generated in-phase carrier $c_{r, i}(t)$ is defined as

$$
c_{r, i}(t)=A_{r} \sin \left(2 \pi f_{c} t\right) \text {. }
$$

The transmitted symbols $s_{i}(t)$ and $s_{q}(t)$ (resp. in-phase and quadrature component) are defined as

$$
\begin{gathered}
s_{i}(t)=\left\{A_{i} \sin \left(2 \pi f_{c} t\right), \quad t=\left\{-T_{g t} / 2, T_{o p}+T_{g t} / 2\right\},\right. \\
s_{q}(t)=\left\{A_{q} \cos \left(2 \pi f_{c} t\right), \quad t=\left\{-T_{g t} / 2, T_{o p}+T_{g t} / 2\right\},,\right.
\end{gathered}
$$

where $A_{i}$ and $A_{q}$ are chosen from interval $\left\{-A_{\max }, A_{\max }\right\}$. A guard time $T_{g t}$ is assumed to be included in the symbol to improve robustness against symbol transition effects [3]. The following analysis is valid for $\tau=\left\{-T_{g t} / 2, T_{g t} / 2\right\}$.

\section{Auto- and Cross-correlations}

The receiver integrates over the interval $t=\left\{0, T_{o p}\right\}$. We calculate the (normalized) autocorrelation $z_{i}(\tau)$ between $c_{r, i}(t)$ and $s_{i}(t)$ :

$$
z_{i}(\tau)=\frac{2}{T_{o p}} \int_{0}^{T_{o p}} s_{i}(t-\tau) \cdot c_{r, i}(t) d t=A_{i} A_{r} \cos \left(2 \pi f_{c} \tau\right),
$$

and the crosscorrelation $z_{q}(\tau)$ between $c_{r, i}(t)$ and $s_{q}(t)$ :

$$
z_{q}(\tau)=\frac{2}{T_{o p}} \int_{0}^{T_{o p}} s_{q}(t-\tau) \cdot c_{r, i}(t) d t=A_{q} A_{r} \sin \left(2 \pi f_{c} \tau\right) .
$$

Mutatis mutandis these calculations (for the in-phase carrier) deliver similar results for the quadrature carrier $c_{r, q}(t)$. In fig. 3, example correlations are shown $\left(A_{i}=A_{q}=A_{\max }\right)$. The units on the x-axis are $\tau / T_{c}$, where $T_{c}=1 / f_{c}$. It can be noticed that there are only few points where the auto-correlation is exactly maximum and the crosscorrelation is exactly zero, and vice versa. The maximum auto-correlation point is (by definition) found at $\tau=0$ (optimum match between transmitter and receiver).
However, at $\tau=T_{c} / 4$ the cross-correlation is maximum. It can be seen that the time shift between the local carrier and the received signal is very critical for optimum reception.

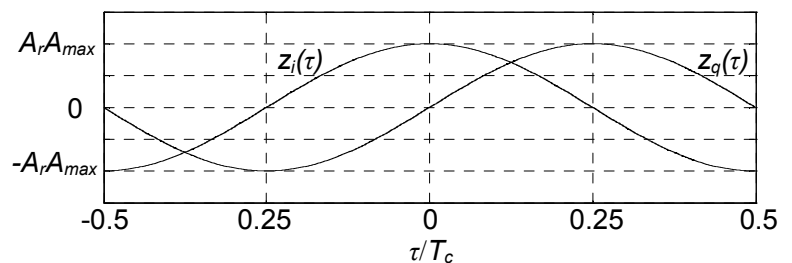

Fig. 3. Autocorrelation $z_{i}(\tau)$ and crosscorrelation $z_{q}(\tau)$ for $A_{i}=A_{q}=A_{\max }$

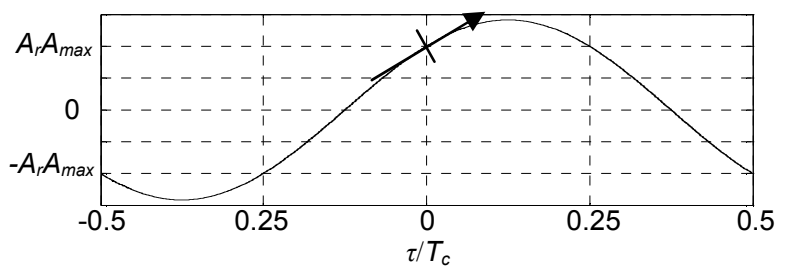

Fig. 4. Correlation $z_{i q}(\tau)$ with summed signals, and derivative at optimum detection point (arrow) for $A_{i}=A_{q}=A_{\max }$

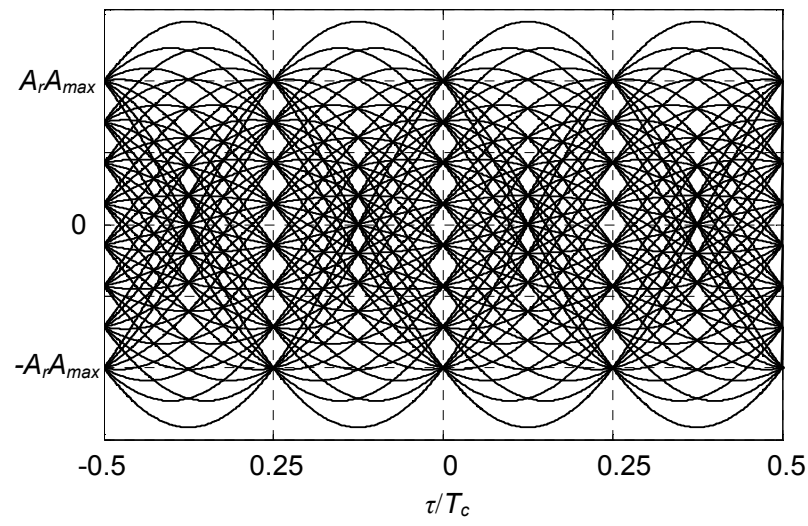

Fig. 5. Correlations $z_{i q}(\tau)$ for all possible combinations of $A_{i}$ and $A_{q}$

Focusing on detection of the in-phase component, we calculate the correlation $z_{i q}(\tau)$ of $c_{r, i}(t)$ with the summed transmitted signal as

$$
\begin{aligned}
z_{i q}(\tau)= & \frac{2}{T_{o p}} \int_{0}^{T_{o p}}\left(s_{i}(t-\tau)+s_{q}(t-\tau)\right) \cdot c_{r, i}(t) d t \\
& =A_{r}\left(A_{i} \cos \left(2 \pi f_{c} \tau\right)+A_{q} \sin \left(2 \pi f_{c} \tau\right)\right) .
\end{aligned}
$$

This is shown in fig. 4 (for $A_{i}=A_{q}=A_{\max }$ ) together with the derivative at the optimum detection point.

Next, we calculate $z_{i q}(\tau)$ for all possible combinations of $A_{i}=\left\{-A_{\max }, A_{\max }\right\}$ and $A_{q}=\left\{-A_{\max }, A_{\max }\right\}$. Plotting all these correlations on top of each other looks a bit like a normal eye diagram. In fig. 5 an example is shown where 3 bits are modulated on both the in-phase and quadrature carrier, resulting in 8 possible levels.

Fig. 5 resembles an eye diagram but it is not the same. Like an eye diagram, these plots can actually be used, in a 
very similar way, to find the optimum detection moment and to analyze the effect of amplitude and time errors on symbol error rate. However, note that the $\mathrm{x}$-axis is not time but relative time shift between $r(t)$ and $c_{r, i}(t)$, in units of $\tau / T_{c}$. The figure shows the effect of a time shift (away from the optimum detection point) on the integrator output.

The impact of a time shift depends on the steepness $y(\tau)$ of the lines around the optimum detection point. We need to calculate this steepness to be able to translate jitter into effective amplitude variation. The steepness is calculated as:

$$
y(\tau)=\frac{d}{d t}\left(z_{i q}(\tau)\right)=2 \pi f_{c} A_{r}\left(A_{q} \cos \left(2 \pi f_{c} \tau\right)-A_{i} \sin \left(2 \pi f_{c} \tau\right) .\right.
$$

For $\tau=0, y(\tau)$ is completely determined by $A_{r} A_{q}$, so it can take on $l$ discrete values, where $l$ is the number of levels used in modulation. To be able to translate from time jitter into worst-case amplitude deviation, we calculate the maximum absolute steepness of these lines $y_{\max }$ as

$$
y_{\max }=\max \left(|y(\tau)|_{\tau=0} \mid\right)=2 \pi f_{c} A_{r} A_{\max } .
$$

(Using [4] it can be shown that the jitter accumulation during the integration period is negligible for $\kappa \sqrt{ } T_{o p} \ll T_{c}$, where $\kappa$ is an oscillator figure of merit.)

\section{Probability of symbol error}

In this section, a 'per-tone symbol error rate' $P_{e}$ is calculated. A 'tone error' occurs when either the in-phase component or the quadrature component of that specific tone is detected incorrectly. The methodology is as follows:

- calculate effective standard deviation of amplitude of integrator output $\left(\sigma_{\text {Aeq }}\right)$ as a function of jitter standard deviation $\left(\sigma_{t}\right)$,

- calculate SNR per symbol from $\sigma_{A e q}$ and distance between levels,

- calculate $P_{e}$ using cumulative normal distribution function.

The total system error rate will be limited by the worst performing tone, so the system should be designed to have an equal error rate for each tone. It is assumed that the jitter coming from the PLL has a Gaussian time distribution with an rms standard deviation of $\sigma_{t}$. Its size is determined by the PLL noise and loop bandwidth. For a well-designed LCbased PLL in the GHz range, currently the rms jitter can be as low as $\sigma_{t}=1 \mathrm{ps}$.

The receiver will compare the integrator output to a number of $(l-1)$ thresholds that are placed in between the amplitude levels. In order to calculate the error rate, we need to calculate the probability that the received signal crosses the threshold between two amplitude levels. In fig. 6, this is illustrated; $t_{x}$ are the thresholds and $s_{x}$ the signal points.

The worst-case effective amplitude standard deviation $\sigma_{A e q}$ as a function of the jitter standard deviation is

$$
\sigma_{A_{e q}}=y_{\max } \sigma_{t}=2 \pi f_{c} A_{r} A_{\max } \sigma_{t}
$$

We can express the distance between levels $2 d$ as a function of $A_{r} A_{\max }$ as

$$
2 d=\frac{2 A_{r} A_{\max }}{l-1}
$$

The error rate is a function of $d / \sigma_{A e q}$. Expressing $d / \sigma_{A e q}$ in terms of $f_{c}, \sigma_{t}$ and $l$ gives

$$
\frac{d}{\sigma_{A_{e q}}}=\frac{1}{2 \pi \sigma_{t} f_{c}(l-1)} .
$$

Next we calculate $P_{i}$, the error rate for the in-phase component, taking into account a factor $(l-1) / l$ because the uppermost and lowermost levels have only one neighbor:

$$
P_{i}=\frac{(l-1)}{l} \frac{1}{\sqrt{2 \pi}} \int_{\frac{d}{\sigma_{A}}}^{\infty} e^{\left(-\frac{y^{2}}{2}\right)} d y=\frac{(l-1)}{l} Q\left(\frac{d}{\sigma_{A_{e q}}}\right)
$$

where $Q(x)$ is the cumulative normal distribution function. Substituting (12) into (13) leads to

$$
P_{i}=\frac{(l-1)}{l} Q\left(\frac{1}{2 \pi \sigma_{t} f_{c}(l-1)}\right)
$$

The probability of error $P_{q}$ for the quadrature component is equal to $P_{i}$. The (total) probability of a tone error $P_{e}$ is

$$
P_{e}=1-\left(1-P_{i}\right)^{2} \text {. }
$$

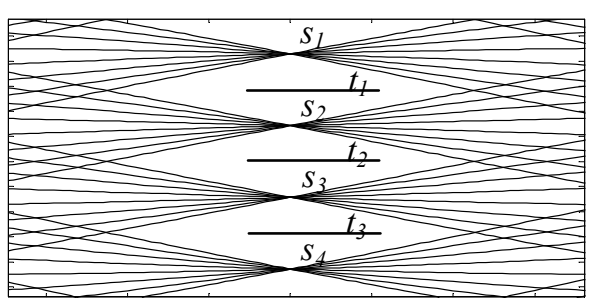

Fig. 6. Amplitude levels and thresholds

Now we can plot $P_{e}$ (at a given $\sigma_{t}$ ) as a function of $f_{c}$ for a number of different modulation depths $n_{b}\left(={ }^{2} \log (l)\right.$, where $n_{b}$ is expressed in bits). This is shown in fig. 7 for $\sigma_{t}=1 \mathrm{ps}$. If necessary, we can convert from symbol to bit errors, e.g. assuming the use of Gray code, so that one symbol error will imply one bit error.

The error rate caused by jitter is a function of modulation depth $n_{b}$ and carrier frequency $f_{c}$. The number of bits that can be modulated onto a carrier (for a given error rate) is limited by jitter, with higher frequency carriers being able to carry fewer bits. In an optimum multi-carrier system, higher frequency carriers should have fewer constellation points to 
achieve the same error rate. This corresponds with results in [2]. In addition, statistical simulations have been done which confirm SNR accuracy of the analysis to within $4 \mathrm{~dB}$ margin.

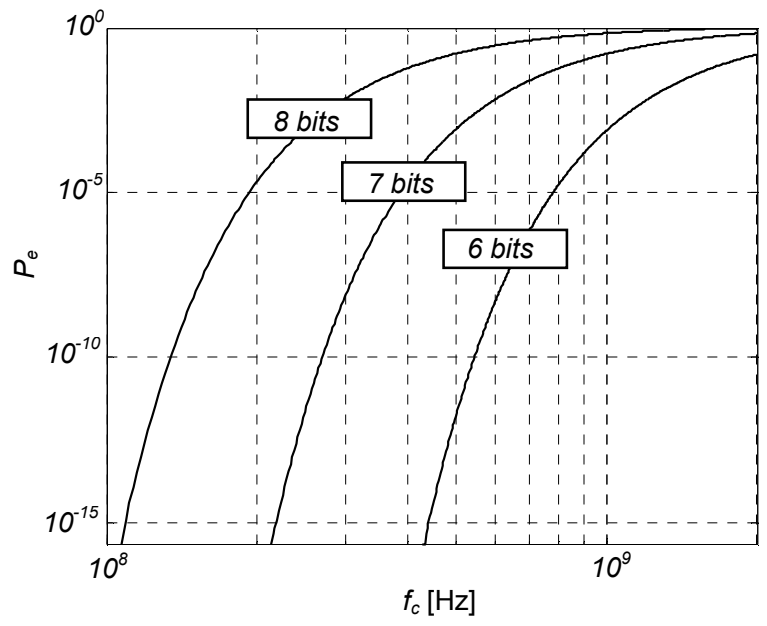

Fig. 7. Probability of error vs. carrier frequency for $\sigma_{t}=1 \mathrm{ps}$

\section{E. Bit rate limits}

The [max. number of bits that can be modulated] $n_{b, \max }$ has been calculated as a function of $\sigma_{t}, f_{c}$ and $P_{e}$, using a numeric solver on (14). Fig. 8 shows the outcome for three different values of $\sigma_{t}=\{0.1 \mathrm{ps}, 1 \mathrm{ps}, 10 \mathrm{ps}\}$, which corresponds to \{excellent, good, fair $\}$, for $P_{e}=1 \cdot 10^{-12}$.

It is interesting to see what the jitter limited maximum bit rate of such a multi-carrier system could be. This is then compared to a PAM system with an equal bandwidth and error rate. In [1] a PAM system is described that can achieve a bit rate of $\sim 7 \mathrm{~Gb} / \mathrm{s}$ for an error probability of $\sim 1 \cdot 10^{-12}$, with a bandwidth of $2 \mathrm{GHz}$ and an rms jitter of $4 \mathrm{ps}$. In our analysis, the upper bound on the multi-carrier system's bit rate is found by integration of $n_{b, \max }$ over a $2 \mathrm{GHz}$ bandwidth and multiplying by two (because both in-phase and quadrature component are used). This delivers a bit rate limit of $14 \mathrm{~Gb} / \mathrm{s}$ (for $\sigma_{t}=4 \mathrm{ps}$ and $P_{e}=1 \cdot 10^{-12}$ ).

The bit rate limit calculated for the multi-carrier system is higher than for the PAM system in [1], but it will have to be corrected downwards for practical implementations due to insertion of a guard time in the symbol. Furthermore, in an implementation with simple switching mixers, a sine wave on the local oscillator port will generate a square wave on the output. This will produce harmonics that fall onto other tone frequencies, creating unusable areas in the spectrum. Next, in case of frequency- or duty-cycle mismatch, inter-carrier interference with carriers at other frequencies will arise. It can be proven that duty-cycle deviations of $>5 \%$ in the receiver generated carrier already cause $P_{e}>1 \cdot 10^{-6}$. From the above it is clear that there is no spectacular improvement in bit rate to be expected from a $\mathrm{GHz}$ multi-carrier system at current state-of-the-art rms jitter figures. Unless properties like robustness against spectral nulls are an important issue, the timing sensitivities make such a system unattractive.

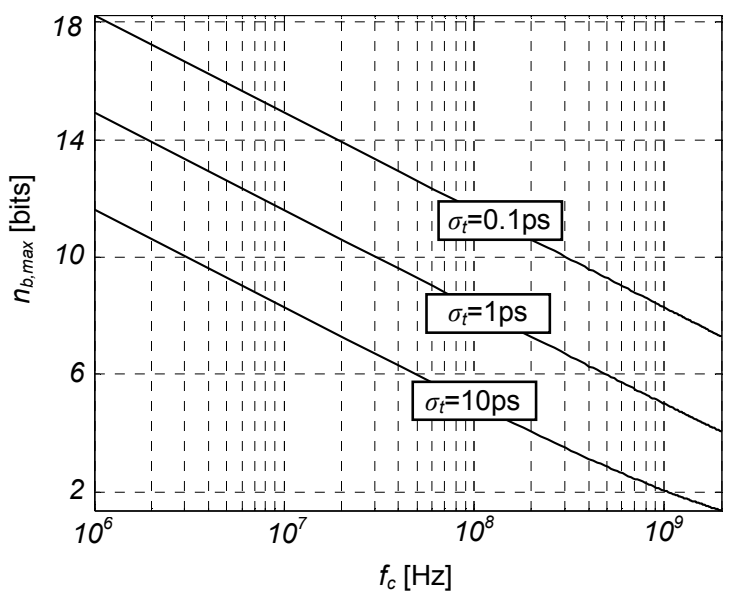

Fig. 8. Max. no. of bits that can be modulated vs. car. freq. for $P_{e}=1 \cdot 10^{-12}$

\section{CONCLUSION}

The feasibility of a $\mathrm{Gb} / \mathrm{s}$ analog OFDM system is analyzed. Correlation plots are introduced to analyze the impact of jitter (coming e.g. from the PLL) on such a multicarrier system. Jitter causes crosstalk between the in-phase and quadrature channels. The maximum bit rate which can be achieved, given a certain specification for the symbol error rate, is limited by this jitter. Assuming a certain rms jitter specification, where the jitter has a Gaussian distribution, it is concluded that low frequency tones can carry more bits than higher frequency tones for the same error rate. Using correlation plots this can be understood in an intuitive way. A jitter limit on the system bit rate is calculated by integrating the area under the plot of [maximum number of bits that can be modulated] versus carrier frequency. The expectations of high spectral efficiencies will not be fulfilled because of the system's high sensitivity to timing errors. It seems that traditional Pulse Amplitude Modulation (PAM) systems with a comparable bandwidth still have the better cards.

\section{ACKNOWLEDGEMENTS}

The authors would like to thank Wim van Etten for helpful discussions.

\section{REFERENCES}

[1] R. Farjad-Rad, C. K. Yang, M. Horowitz, and T. H. Lee, "A 0.3- $\mu \mathrm{m}$ CMOS 8-Gb/s 4-PAM serial link transceiver," IEEE J. Solid-State Circuits, vol. 35, pp. 757-764, May 2000.

[2] T. N. Zogakis and J. M. Cioffi, "The effect of timing jitter on the performance of a discrete multitone system," IEEE Trans. Commun., vol. 44, pp. 799-808, July 1996.

[3] J. A. C. Bingham, "Multicarrier modulation for data transmission: An idea whose time has come," IEEE Commun. Mag., vol. 28, pp. 5-14, May 1990.

[4] J. McNeill, "Jitter in ring oscillators," IEEE J. Solid-State Circuits, vol. 32, pp. 870-879, June 1997. 